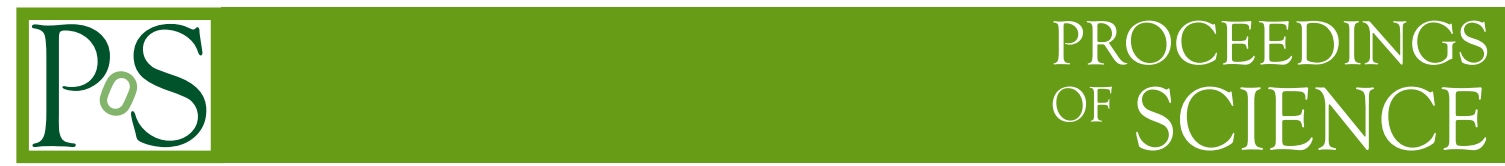

\title{
Searches for dark matter at CMS in events with missing transverse energy
}

\author{
Christian Laner* on behalf of the CMS Collaboration \\ Imperial College (GB) \\ E-mail: c.lanerecern.ch
}

\begin{abstract}
Many theories predict candidates to the dark matter particles that are light enough to be produced at the LHC. Various searches for dark matter at CMS in events with missing transverse energy using up to $12.9 \mathrm{fb}^{-1}$ of data are presented. The final states considered include jets, $\mathrm{W}$ and $\mathrm{Z}$ bosons, top and bottom quarks, and Higgs bosons. Limits are set on various simplified models.
\end{abstract}

25th International Workshop on Deep Inelastic Scattering and Related Topics

3-7 April 2017

University of Birmingham, Birmingham, UK

\footnotetext{
* Speaker.
} 


\section{Introduction}

Dark matter (DM) constitutes about $26 \%$ of the total energy content of the universe. Its nature is not explained by the standard model and its presence has so far only been inferred indirectly through its gravitational effects. The most favoured DM candidate is a non-baryonic, weakly interacting massive particle (WIMP) which is stable and electrically neutral.

WIMPs may be produced in the high energy proton-proton collisions at the CERN LHC. Although they would not be detectable, they may recoil against a standard model object $X$ and result in an apparently momentum-unbalanced event. Its presence would be inferred by missing transverse energy $\left(E_{\mathrm{T}}^{\text {miss }}\right)$, which is the negative vector sum of the observable particles' transverse energies. Searches for DM at the LHC are complimentary to direct searches that look for DMnucleus scattering and indirect searches that look for DM annihilation.

The dark matter searches at CMS [1] presented here consider $E_{\mathrm{T}}^{\text {miss }}+X$ final states with $X$ being a jet, vector boson, photon, heavy quark, or Higgs boson. The results are interpreted using the simplified models developed in Ref. [2]. These models assume fermionic dark matter that is pair-produced in an s-channel via a vector, axial-vector, scalar, or pseudoscalar mediator. The minimal set of parameters included are the masses of the DM and mediator, as well as the coupling strengths of the mediator to SM and DM particles.

\section{2. $E_{\mathrm{T}}^{\mathrm{miss}}+$ jet/hadronic-W/Z [3]}

The $E_{\mathrm{T}}^{\text {miss }}+$ jets final state is the one with the largest production cross section. Candidate events are selected by requiring a large amount of $E_{\mathrm{T}}^{\text {miss }}(>200 \mathrm{GeV})$ and at least one highly energetic jet $(>100 \mathrm{GeV})$. Events containing leptons, photons, or b-tagged jets are vetoed.

$\mathrm{A} E_{\mathrm{T}}^{\mathrm{miss}}+\mathrm{W} / \mathrm{Z}$ category is also considered in order to capture final states containing a hadronically decaying $\mathrm{W}$ or $\mathrm{Z}$ boson. The vector boson tends to be quite boosted and so the two resulting hadronic products appear as a single "fat" jet, which is reconstructed using the anti- $\mathrm{k}_{\mathrm{T}}$ algorithm using a distance parameter of 0.8 instead of the usual value of 0.4 .

The dominant background processes are $\mathrm{Z}+$ jets with the $\mathrm{Z}$ boson decaying to neutrinos, and $\mathrm{W}+$ jets with the $\mathrm{W}$ boson decaying leptonically and the lepton not being identified or falling outside of the acceptance of the detector. A precise estimation of these backgrounds is required, and this is achieved using five control regions consisting of muon, di-muon, electron, di-electron, and photon final states. A maximum likelihood fit is performed to the $E_{\mathrm{T}}^{\text {miss }}$ distribution over all $E_{\mathrm{T}}^{\text {miss }}+$ jet and $E_{\mathrm{T}}^{\text {miss }}+\mathrm{W} / \mathrm{Z}$ control and signal regions in order to estimate the background events and extract the signal strength. The result for the $E_{\mathrm{T}}^{\text {miss }}+$ jet category is shown in Fig. 1.

\section{3. $E_{\mathrm{T}}^{\text {miss }}+$ photon $[4]$}

The $E_{\mathrm{T}}^{\text {miss }}+$ photon final state has a smaller cross section than the $E_{\mathrm{T}}^{\text {miss }}+$ jet final state but provides a cleaner search channel as the amount of background is smaller.

Candidate events are selected by requiring one central highly energetic photon $(>175 \mathrm{GeV})$, a large amount of $E_{\mathrm{T}}^{\text {miss }}(>170 \mathrm{GeV})$. Analagously to the $E_{\mathrm{T}}^{\text {miss }}+$ jet channel, the dominant background processes are $\mathrm{Z}(v v)+\gamma$ and $\mathrm{W}(\ell v)+\gamma$. In this case, the potential control regions are more 
statistically limited, and so the backgrounds are estimated with Monte Carlo simulation corrected for NLO electroweak and NNLO QCD contributions.

The $E_{\mathrm{T}}^{\text {miss }}$ distribution for the selected events are show in Fig. 1 together with the estimated background processes.
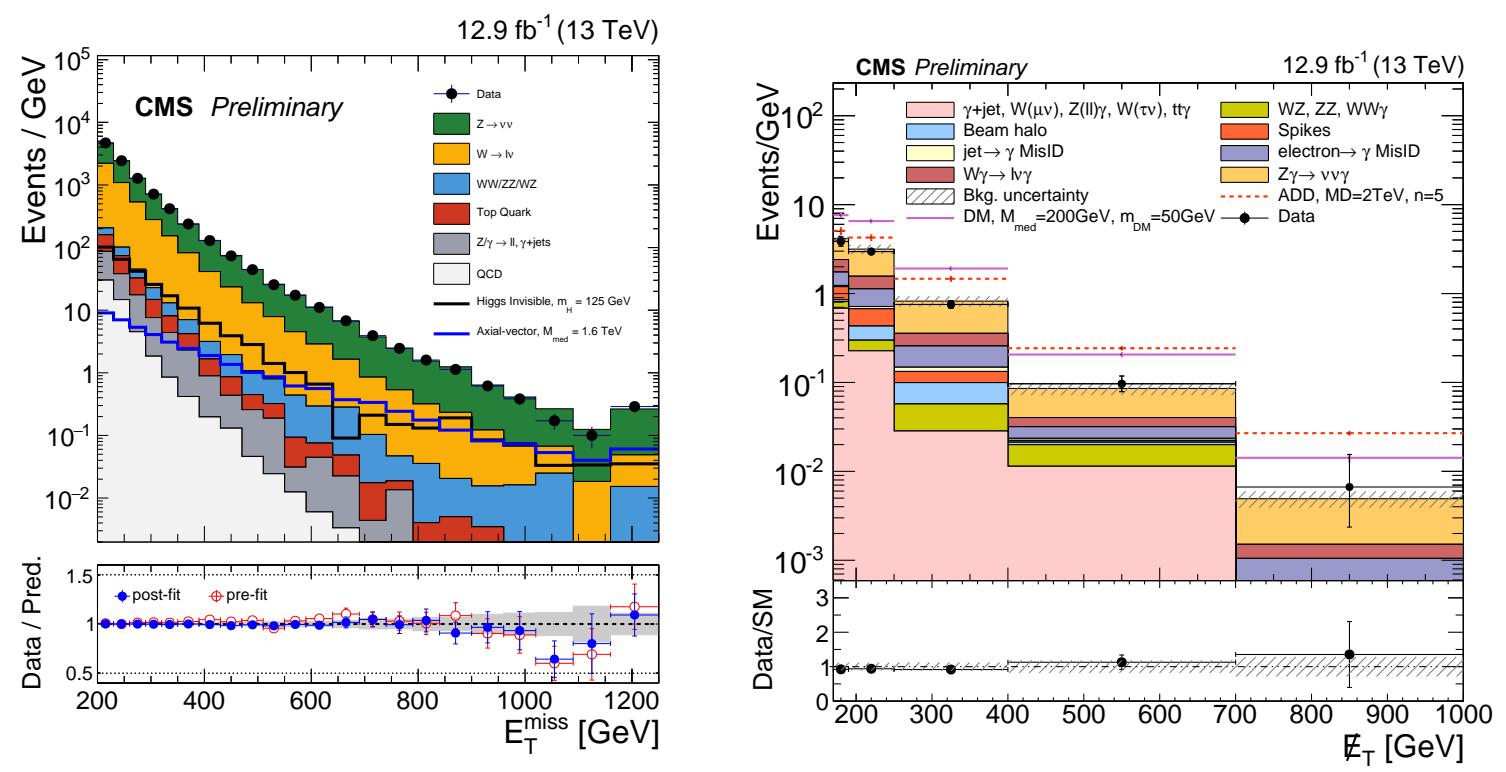

Figure 1: Observed $E_{\mathrm{T}}^{\text {miss }}$ distribution in the signal region for the $E_{\mathrm{T}}^{\text {miss }}+$ jets (Left) and $E_{\mathrm{T}}^{\text {miss }}+$ photon (Right) searches compared to the estimated contributions from standard model background processes.

\section{4. $E_{\mathrm{T}}^{\mathrm{miss}}+$ leptonic-Z [5]}

In this channel, events containing two opposite-sign, same-flavour leptons (muons or electrons) with an invariant mass consistent with the $\mathrm{Z}$ boson mass are selected.

The dominant background processes in this case are diboson events, namely $\mathrm{Z}(\ell \ell) \mathrm{Z}(v v)$ and $\mathrm{Z}(\ell \ell) \mathrm{W}(\ell v)$. These are estimated using Monte Carlo simulation with higher order corrections applied. The analyis is performed via maximum likelihood fit to the $E_{\mathrm{T}}^{\text {miss }}$ distribution.

\section{5. $E_{\mathrm{T}}^{\mathrm{miss}}+\mathrm{t} \overline{\mathrm{t}} / \mathrm{b} \overline{\mathrm{b}}[6]$}

If the SM-DM mediator is of spin 0, it may couple to quarks with a Yukawa-like structure, and hence couple preferentially to heavy quarks, namely top and bottom quarks. The dark matter would be produced in association with top or bottom quark pairs. Four channels are considered in this search.

The hadronic t⿱亠 final state, in which both top quarks decay hadronically, is the one with the largest cross section. A multivariate top-tagging algorithm is employed in order to identified the three hadronic products of the top quark. Events are categorised according to the number of identified top-tags to help discriminate between signal and background. 
The semileptonic t $\bar{t}$ final state consists of one hadronically decaying top quark and another decaying semileptonically. The dileptonic $t \bar{t}$ channel consists of two semileptonically decaying top quarks.

Finally, the $b \bar{b}$ final state is considered in order to capture the DM production in association with bottom quark pairs, as well as to capture any $t \bar{t}$ associated production not captured by the three previous $\bar{t} \bar{t}$ channels.

The final results are obtained by performing a simultaneous fit over all four channels, with the $\mathrm{t} \overline{\mathrm{t}}$ and $\mathrm{b} \overline{\mathrm{b}}$ signal processes combined.

6. $E_{\mathrm{T}}^{\mathrm{miss}}+\mathbf{t}[7]$

The simplified model for the $E_{\mathrm{T}}^{\text {miss }}+\mathrm{t}$ final state consists of the single top quark being produced via a flavour-changing vector current.

Evets with $E_{\mathrm{T}}^{\text {miss }}>250 \mathrm{GeV}$ and a boosted hadronic top quark are selected. The top-tagging is performed with a jet-clustering cone size of 1.5, and with mass and substructure variables that are required to be consistent with a three-prong hadronic decay of a top quark. The dominant background processes, as well as the estimation of these and the extraction of signal proceeds in a similar way to the $E_{\mathrm{T}}^{\text {miss }}+$ jets analysis.

\section{7. $E_{\mathrm{T}}^{\mathrm{miss}}+\mathbf{H}[8,9]$}

If dark matter acquires mass via a Higgs-like mechanism, a possible final state consists of a Higgs boson being produced in association with the DM. The radiation of a Higgs boson is Yukawa-suppressed, but one may consider a two-Higgs-doublet model.

Two different final states are considered, in which the Higgs boson decays to a pair of bottom quarks or a pair of photons. A large amount of $E_{\mathrm{T}}^{\mathrm{miss}}$ and an invariant mass of the final state system consistent with the Higgs boson mass are required. In the b $\bar{b}$ search, both a resolved regime, in which the two $b$ quarks form distinct jets, and a boosted regime, in which the two b quarks appear as a single "fat" jet, are considered.

\section{Interpretation of results}

No significant deviation from the standard model expectation is observed, and so the results are interpreted by setting upper limits on various simplified dark matter models.

Figure 2 shows $95 \%$ confidence level (CL) limits as a function of DM and mediator masses, for models with a spin-1 (vector or axial-vector) mediator with coupling strenghts of $g_{q}=0.25$ and $g_{\mathrm{DM}}=1$. The most sensitive channel, $E_{\mathrm{T}}^{\text {miss }}+$ jets, is excluding mediator masses of up to $2 \mathrm{TeV}$ and DM masses of over 700 (500) GeV for the vector (axial-vector) model.

These limits are translated to $90 \%$ CL limits on the DM-nucleon scattering cross section in Fig. 3, and compared to the results of direct detection searches. The collider constraints are particularly complementary for low DM masses $(<5 \mathrm{GeV})$ in the spin-independent scenario, and for a wide range of $\mathrm{DM}$ masses $(<600 \mathrm{GeV})$ in the spin-dependent scenario. 
Finally, Fig. 4 shows 95\% confidence level limits on models with a spin-0 (scalar or pseudoscalar) mediator with coupling strengths of $g_{q}=g_{\mathrm{DM}}=1$. The lowest masses of these models are just starting to become excluded with the data set at hand.
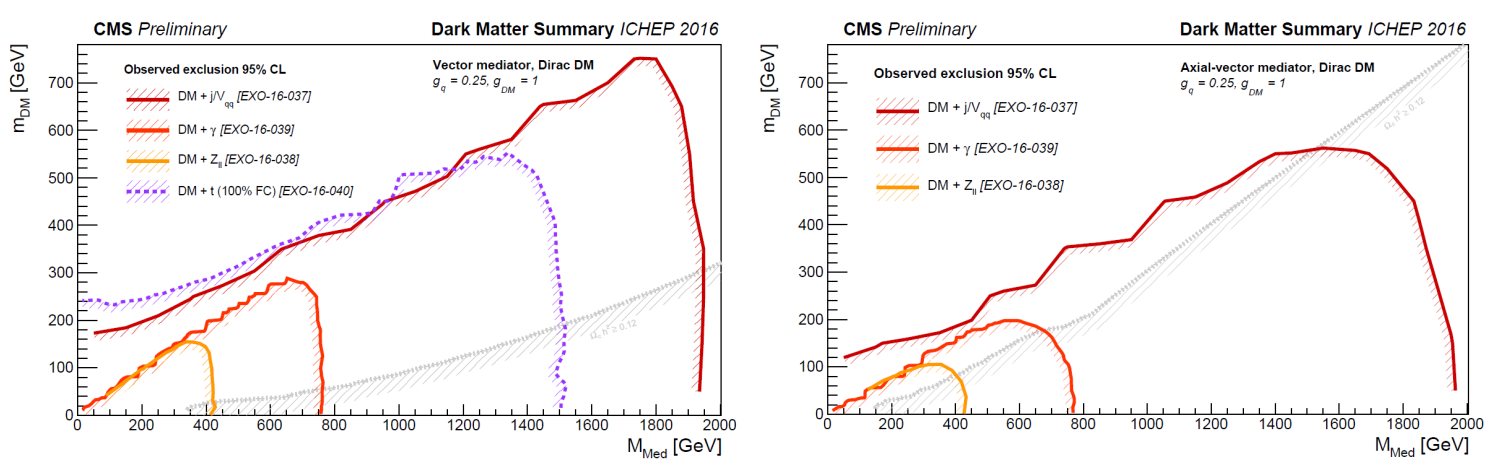

Figure 2: $95 \%$ CL exclusion regions as a function of mediator and DM masses for various $C M S E_{\mathrm{T}}^{\text {miss }}$ based DM searches for vector (Left) and axial-vector (Right) models [10].
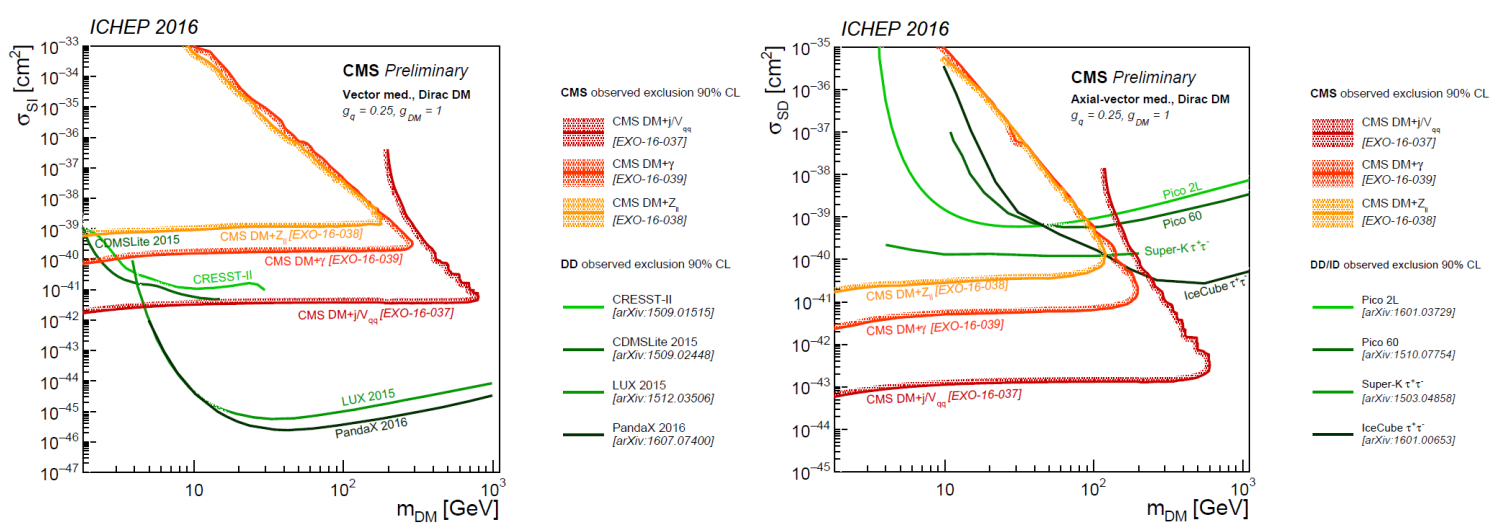

Figure 3: $90 \%$ CL exclusion regions on the spin-independent (Left) and spin-dependent (Right) DMnucleon scattering cross section as a function of DM mass for various CMS $E_{\mathrm{T}}^{\text {miss }}$ based searches compared to direct and indirect detection experiments [10].

\section{Summary}

Searches for dark matter have been perfromed in various $E_{\mathrm{T}}^{\mathrm{miss}}+\mathrm{X}$ final states at CMS using 2.2 or $12.9 \mathrm{fb}^{-1}$ of $\sqrt{s}=13 \mathrm{TeV}$ collision data. No excess above the SM expectation has been observed and limits are set on a variety of simplified models. LHC searches have been seen to be complimentary to direct and indirect detection experiments.

\section{References}

[1] CMS Collaboration, The CMS experiment at the CERN LHC, JINST 3 S08004 (2008)

[2] D. Abercrombie et al., Dark Matter Benchmark Models for Early LHC Run-2 Searches: Report of the ATLAS/CMS Dark Matter Forum, arXiv:1507.00966 

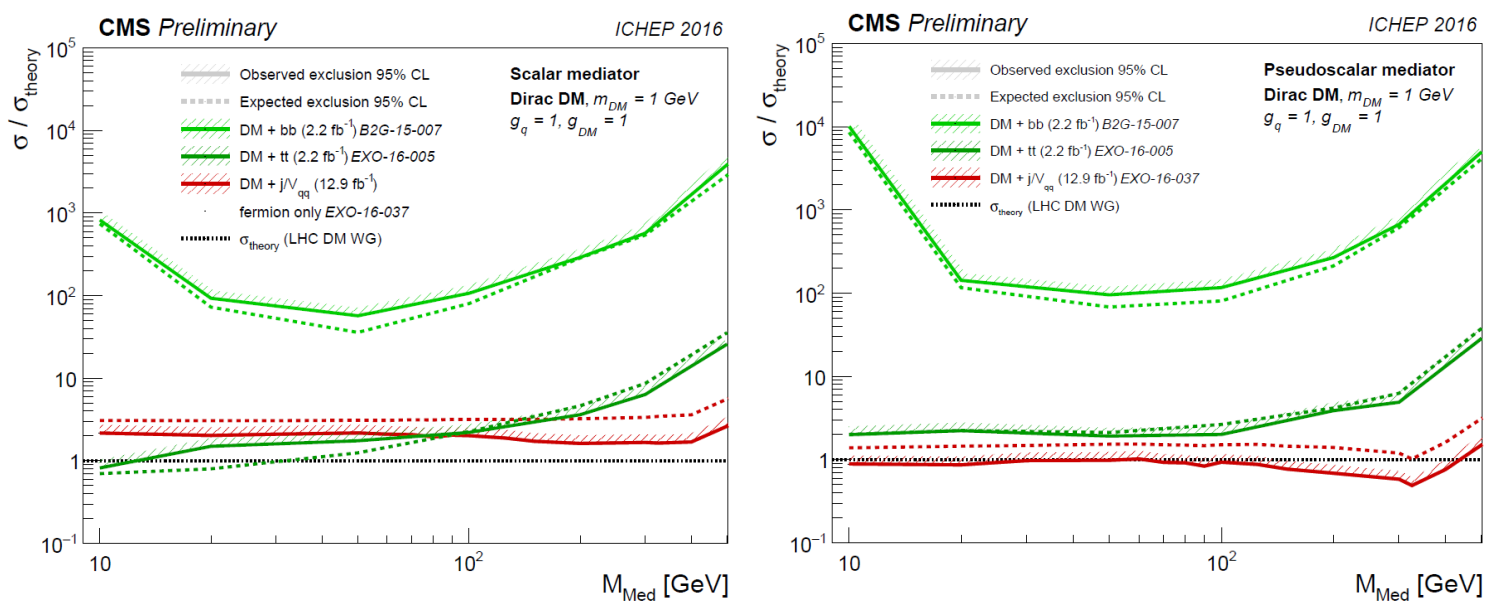

Figure 4: 95\% CL exclusion regions as a function of mediator and DM masses for various CMS $E_{\mathrm{T}}^{\text {miss }}$ based DM searches for scalar (Left) and pseudoscalar (Right) modelsi [10].

[3] CMS Collaboration, Search for dark matter in final states with an energetic jet, or a hadronically decaying $W$ or $Z$ boson using $12.9 \mathrm{fb}^{-1}$ of data at $\sqrt{\mathrm{s}}=13 \mathrm{TeV}$, CMS-PAS-EXO-16-037

[4] CMS Collaboration, Search for dark matter and graviton produced in association with a photon in pp collisions at $\sqrt{\mathrm{s}}=13 \mathrm{TeV}$ with an integrated luminosity of $12.9 \mathrm{fb}^{-1}$, CMS-PAS-EXO-16-039

[5] CMS Collaboration, Search for dark matter in $Z+E_{\mathrm{T}}^{\mathrm{miss}}$ events using $12.9 \mathrm{fb}^{-1}$ of 2016 data, CMS-PAS-EXO-16-038

[6] CMS Collaboration, Search for dark matter in association with a top quark pair at $\sqrt{s}=13 \mathrm{TeV}$, CMS-PAS-EXO-16-005

[7] CMS Collaboration, Search for new physics in a boosted hadronic monotop final state using 12.9 $\mathrm{fb}^{-1}$ of $\sqrt{\mathrm{s}}=13 \mathrm{TeV}$ data, CMS-PAS-EXO-16-040

[8] CMS Collaboration, Search for Dark Matter Produced in Association with a Higgs Boson Decaying to Two Photons, CMS-PAS-EXO-16-011

[9] CMS Collaboration, Search for dark matter in association with a Higgs boson decaying into a pair of bottom quarks at $\sqrt{s}=13 \mathrm{TeV}$ with the CMS detector, CMS-PAS-EXO-16-012

[10] CMS Collaboration, Dark Matter Summary Plots from CMS for ICHEP 2016, CMS-DP-2016-057 